\title{
Clinical outcomes of switching to aflibercept using a pro re nata treatment regimen in patients with neovascular age-related macular degeneration who incompletely responded to ranibizumab
}

Flora Elwes', Shyamanga Borooah ${ }^{2,4^{*}}$, Peter Aspinall ${ }^{3,4}$, Peng Yong Sim ${ }^{4}$, Cheng Yi Loo ${ }^{4}$, Ana-Maria Armbrecht ${ }^{2}$, Baljean Dhillon ${ }^{2,4}$ and Peter Cackett ${ }^{2,4}$

\begin{abstract}
Background: To assess the effect of switching patients previously incompletely treated with ranibizumab (RBZ) to aflibercept (AFL) using a pro re nata (PRN) treatment strategy in neovascular age-related macular degeneration (nvAMD).

Methods: A retrospective case series was conducted on patients who had persistent or recurrent intra- and/or sub-retinal fluid treated initially with RBZ and subsequently switched to AFL. The main outcome measures were best corrected visual acuity (BCVA) and central retinal thickness (CRT) measured at different stages of the study. Friedman analysis of variance and Wilcoxon test were used to examine differences in BCVA and CRT.

Results: Two hundred and seven eyes from 182 patients were included. BCVA and CRT improved significantly initially following 3 RBZ injections with a mean gain of 3.7 letters $(p<0.001)$ and a mean loss of $69 \mu \mathrm{m}(p<0.001)$ respectively. Following PRN RBZ therapy and immediately prior to switching to AFL (mean 129 weeks), there was a mean loss of 6.7 letters $(p<0.001)$ BCVA and a mean gain of $24 \mu \mathrm{m}(p<0.001)$ CRT.

AFL loading resulted in a mean improvement of 0.7 letters $(p=0.28)$ BCVA and $55 \mu m(p<0.001)$ CRT. At final follow-up following AFL PRN therapy (mean 85 weeks), there was a mean loss of 8.9 letters $(p<0.001)$ BCVA and a mean gain of $12 \mu \mathrm{m}(p<0.05)$ CRT.
\end{abstract}

Conclusion: AFL loading resulted in a significant anatomical improvement but no significant change in visual acuity. However, the benefits of switching were gradually lost over time with AFL PRN dosing despite an increased injection rate when compared with RBZ PRN treatment.

Trial registration: Not applicable

Keywords: Age-related macular degeneration, Ranibizumab, Aflibercept, Anti-VEGF

\footnotetext{
* Correspondence: shyamanga@aol.com

${ }^{2}$ The Princess Alexandra Eye Pavilion, Edinburgh, UK

${ }^{4}$ College of Medicine \& Veterinary Medicine, University of Edinburgh,

Edinburgh, UK

Full list of author information is available at the end of the article
} 


\section{Background}

Neovascular age-related macular degeneration (nvAMD) is one of the leading causes of visual impairment in the population aged over 50 years of age [1]. The prevalence of sight threatening nvAMD is predicted to increase with time [2].

The mainstay of treatment for nvAMD is with intravitreal administration of drugs targeting vascular endothelial growth factor (VEGF). Ranibizumab (RBZ) [Lucentis, Genentech, San Francisco, California, USA] was first licensed by the Federal Drug Administration (FDA) for the treatment of nvAMD in 2006 and became the most widely used anti-VEGF agent. RBZ is a monoclonal antibody fragment that specifically targets VEGF-A [3]. Large multicentre clinical trials have demonstrated that RBZ intravitreal therapy (IVT) stabilises long-term bestcorrected visual acuity (BCVA) in the majority of patients with nvAMD and improves BCVA in a minority of patients $[4,5]$. However, maintaining a frequent treatment schedule for patients with nvAMD is difficult for patients and places a heavy burden on health services [6]. As a result a number of variable treatment, followup and dosing schedules have been developed including pro re nata (PRN) strategies [7-9]. Despite RBZ treatment some patients with nvAMD continue to demonstrate persistent macular fluid [10]. Taken together this points to the need to test alternative treatments for nvAMD in patients who are incompletely treated with RBZ IVT.

Aflibercept (AFL) [Eylea, Regeneron, Tarrytown, New Jersey] was FDA-approved as an alternative anti-VEGF treatment for nvAMD in late 2011. AFL is a recombinant fusion protein consisting of VEGF-binding portions from the extracellular domains of human VEGF receptors. These protein domains are fused to the Fc portion of a human immunoglobulin to improve their half-life [11]. AFL mimics VEGF target receptors and acts to trap both VEGF-A, VEGF-B and placental growth factor reducing downstream effects of these chemokines. Multicentre clinical trials have also confirmed the clinical efficacy of AFL in the treatment of nvAMD [12]. Additionally, AFL has also been shown to reduce persistent macular fluid in cases of nvAMD which appear refractory to treatment with RBZ $[13,14]$. AFL has been found to have a higher binding affinity for VEGF than RBZ which predicts a lower required concentration and potentially longer clinical effect [15]. AFL administered at an interval of two months after 3 initial monthly loading doses, was found to be non-inferior to RBZ in treatment-naive eyes [12]. The less frequent treatment regime with AFL and reduced cost of AFL compared with RBZ treatment for nvAMD also has potential implications for improved cost-utility when compared with RBZ in a PRN dosing schedule [16].
Increasingly patients who have initially been treated with RBZ are being switched to AFL. However, there is a relative dearth of real world clinical data on the effect of switching patients using a PRN treatment strategy. In this study we describe the effects of switching to AFL PRN therapy in AMD patients who had recurrent nvAMD despite a RBZ PRN schedule at a regional AMD treatment centre in the United Kingdom.

\section{Methods \\ Design \& Patients}

A retrospective case series was conducted on patients attending the Princess Alexandra Eye Pavilion, Edinburgh, a tertiary referral centre for nvAMD. A treatment register was maintained of all patients who attended the centre between September 2013 and May 2014 and required ongoing anti-VEGF treatment. From this register we identified consecutive patients who were switched from RBZ to AFL. This therapeutic switch was based on a change in protocol for treatment instituted by the department. Switch patients were followed up prospectively for a minimum of 12 months after switch. Paper-based health records for these patients were retrospectively reviewed between 1 April 2016 and 26 June 2016.

Verbal informed consent for prospective data collection was obtained from all participants at the first injection visit by the treating clinician. The study was given ethical approval, granted a waiver of documentation of informed consent for retrospective analysis and approved for verbal consent with regard to prospective follow-up by the NHS Lothian research ethics committee with approval number 09/S1101/05. All procedures adhered to the tenets of the Declaration of Helsinki.

All inclusion criteria refer to the study eye only. In order to be included in the study, patients had to have a diagnosis of nvAMD as demonstrated by changes on retinal examination, optical coherence tomography (OCT) or fundus fluorescein angiography initially. In addition, to be switched they had to have persistent or recurrent intra- and/or sub-retinal fluid on OCT consistent with active disease at least 6 months after RBZ loading. Patients must also have had a minimum of 4 RBZ injections which comprised the loading phase (3 consecutive monthly injections) followed by at least 1 further injection.

Exclusion criteria for the study eye included bestcorrected visual acuity $\geq 1.3 \log$ MAR as this exceeded local guidelines for treatment with anti-VEGF.

\section{Study protocol}

For all patients, data was collected at 5 intervals representing 4 stages of the study (Fig. 1). In stage 1, patients were reviewed prior to initiating RBZ treatment and 


\section{Data Collection Intervals 1 - 5}

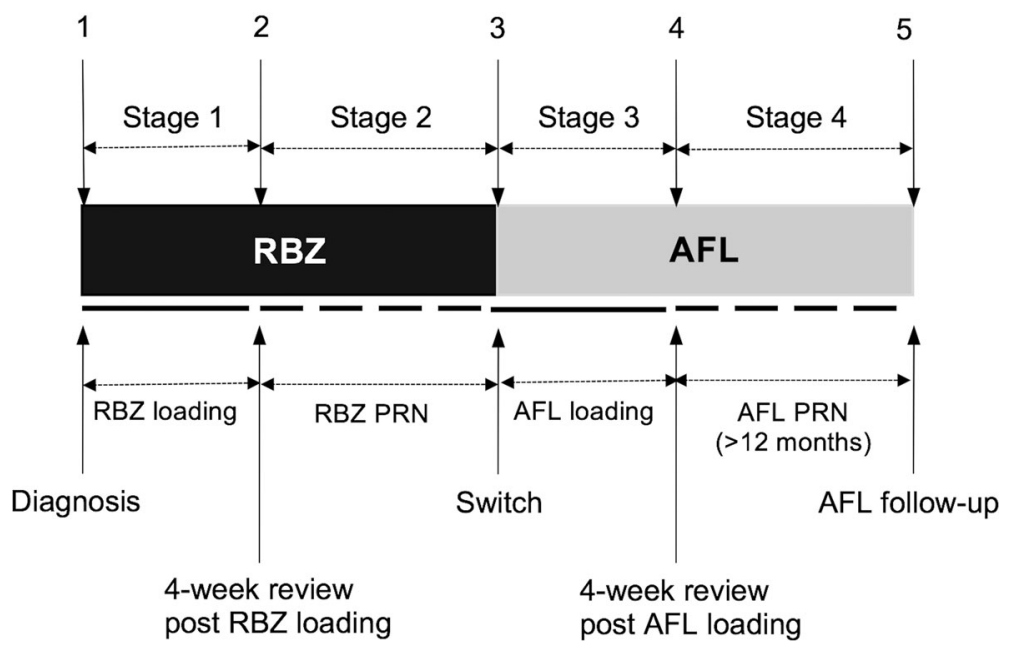

Fig. 1 Diagram summarising the timeline of data collection in this study. All patients had an initial loading phase of 3 monthly injections of RBZ (stage 1) followed by a PRN treatment schedule with RBZ (stage 2) which was on average 129 ( \pm 86 ) weeks. After switch, patients had 3 loading monthly injections of AFL (stage 3) followed by PRN AFL (stage 4). The mean time for AFL PRN treatment was $85( \pm 18)$ weeks. Data was collected at baseline (1) and 4-week review post RBZ loading (2). Further data was collected at switch to AFL (3), 4-week review post AFL loading (4) and > 12 months after switch (5)

4 weeks after the third loading dose of RBZ. In stage 2, patients were then treated with RBZ following a PRN protocol.

At switch, 3 loading doses of intravitreal AFL $(2 \mathrm{mg})$ were administered at approximately 4-week intervals. Stage 3 was from immediately prior to anti-VEGF switch and 4 weeks after the third loading dose of AFL. In stage 4 patient were treated with AFL again on a PRN schedule and followed up after a minimum of 12 months following AFL switch. For both PRN phases, a decision to retreat was made if there was evidence of intra- and/or sub-retinal fluid on SD-OCT.

At all visits, patients underwent a full ophthalmic examination of both eyes, which included best corrected visual acuity (BCVA) measured using Early Treatment Diabetic Retinopathy Study (ETDRS) letters (recorded at $4 \mathrm{~m}$ ) whilst central retinal thickness (CRT) measured using OCT. The same Zeiss Cirrus SD-OCT machine (Carl Zeiss Meditec Inc., Dublin, CA) was used for all CRT measurements. Additionally, intraocular pressures and adverse event monitoring was performed at each visit. Data was also collected on the duration of disease, the number of injections performed and phakic status. If follow-up was deemed appropriate, the schedules were decided by the ophthalmologist depending on activity and varied between 1 and 4 months.

\section{Injection procedure}

All RBZ and AFL injections were performed according to a standardised protocol in a treatment room setting by the same healthcare team. Patients were prepared by administration of topical anaesthesia (Minims tetracaine hydrochloride $0.5 \%$ ), followed by application of a topical antiseptic (povidine iodine $5 \%$ or chlorhexidine $0.1 \%$ in the case of documented prior iodine allergy). The injection was performed using a 30-gauge needle through the pars plana. All patients were given a one-off dose of chloramphenicol ointment following injection.

\section{Statistical analysis}

The analysis was performed in three parts. Firstly, differences in BCVA and CRT were examined using a Friedman analysis of variance followed by Wilcoxon tests for paired comparisons. Secondly, correlations between change in BCVA and CRT were analysed by principle component analysis with varimax rotation. Finally, a correlated component regression analysis examining the predictors of change in visual outcome was carried out at each of the 4 stages of the study [see Additional file 1 for further details of statistical analyses]. All statistical analyses were carried out using SPSS statistic version 17.0 (IBM Corp., Armonk, N.Y., USA).

\section{Results}

\section{Patient characteristics}

A total of 213 eyes from 188 patients were identified from the treatment register; 6 of the 188 patients were excluded due to missing case notes, leaving 207 eyes from 182 patients for analysis. A summary of the baseline details of the participants at switch and duration of 
AMD by switch and number of RBZ injections is shown in Table 1. The mean age at switch was 80 years $( \pm 7.7)$ and $71 \%$ of patients were female. The mean duration of nvAMD in patients was $129( \pm 86)$ weeks at switch. Baseline mean BCVA $( \pm$ SD) was $29( \pm 13.2)$ ETDRS letters whilst the baseline mean CRT $( \pm$ SD) was $342 \mu \mathrm{m}( \pm 100)$.

Patients received monthly RBZ injections for RBZ loading in stage 1 . The mean treatment period during stage $2($ RBZ PRN) was $129( \pm 86)$ weeks with patients receiving an average of $12( \pm 7) \mathrm{RBZ}$ injections over this time.

At switch patients received 3 loading doses of AFL with a month interval. In stage 4 , patients received AFL PRN therapy. 9 patients were lost to follow-up, 7 patients were swapped back to RBZ due to poor response to AFL, and a further 7 patients did not require further anti-VEGF treatment after AFL loading but continued under regular follow-up. These latter two groups of patients are included in the overall analysis as well as a separate analysis within this paper. The average followup for stage 4 of the study was $85( \pm 18)$ weeks with patients receiving an average of $10( \pm 3)$ AFL injections over this time, achieving an average injection frequency of 8.6 weeks.

\section{Visual acuity changes}

BCVA improved significantly following 3 RBZ loading injections, with a mean gain of 3.7 ETDRS letters $(p<$ 0.001 ) when compared to baseline. At switch, BCVA had deteriorated with a mean loss of 6.7 letters $(p<0.001)$. This was a net loss of 3 ETDRS letters from baseline after a mean treatment period of 129 weeks with a mean of 12 injections resulting in a mean injection frequency of an injection every 10.75 weeks.

After AFL loading, the BCVA had increased by a mean of 0.7 EDTRS letters from switch, although this change $(p=0.28)$ was not statistically significant from BCVA at switch. After the AFL PRN treatment phase mean BCVA had declined with a mean loss of 8.9 letters $(p<0.001)$ over a mean treatment phase of $85( \pm 18)$ weeks (Fig. 2).

Table 1 Summary of baseline patient details

\begin{tabular}{ll}
\hline Characteristics & \\
\hline No. of participants & 182 \\
No. of eyes & 207 \\
Mean age (years) \pm SD & $80 \pm 7.7$ \\
Female (\%) & 71 \\
Phakic eyes (\%) & 78 \\
Mean BCVA (letters) \pm SD & $29 \pm 13.2$ \\
Mean central retinal thickness ( $\mu$ m) \pm SD & $342 \pm 100$ \\
Mean duration of nvAMD at switch (weeks) \pm SD & $129 \pm 86$ \\
Mean number of ranibizumab injections at switch \pm SD & $12 \pm 7$ \\
\hline
\end{tabular}

This resulted in an overall mean loss of 11.2 letters from baseline with a total mean treatment time of 229 weeks. The rate of decline of BCVA during RBZ PRN treatment saw an average loss of 3.2 letters per eye per year. In the AFL PRN treatment phase BCVA was reduced on average by 5.5 letters per eye per year. Letters per eye per year was defined as the total sum of letters gained or lost divided by the total number of eyes and the total mean treatment time of the stage examined.

\section{Anatomic changes}

Changes in CRT followed a similar trend to BCVA. CRT decreased by an average of $69 \mu \mathrm{m}(p<0.001)$ following the 3 RBZ loading injections. At switch CRT had increased by a mean of $24 \mu \mathrm{m}(p<0.001)$. Following AFL loading, the CRT showed a significant mean reduction of $55 \mu \mathrm{m}(p<0.001)$. After AFL PRN treatment, the CRT had increased by an average of $12 \mu \mathrm{m}(p<0.05)$ (Fig. 3).

Looking at average changes over time per eye per year for each stage, with RBZ PRN therapy there was an increase in mean CRT of $12 \mu \mathrm{m}$ per eye per year, which was reduced to $7 \mu \mathrm{m}$ per eye per year on AFL PRN.

After AFL loading, 173 eyes (83.6\%) showed static or reduced CRT. However, after a minimum of 12 months follow-up of patients on AFL PRN treatment only 80 eyes had static or reduced CRT (43\%) (Table 2).

A principal component analysis with varimax rotation was carried out to examine the relationship between BCVA and CRT during the study. The results showed that there was a positive correlation between BCVA and CRT change in stage 1 , meaning that better BCVA was associated with reduced CRT during RBZ loading. However, this relationship was not maintained in stages 2,3 or 4 .

To identify predictors of final visual outcome a regression analysis was used to examine the predictors of change in BCVA and CRT at each stage of the study. Better BCVA at the start of stages 1,2 and 3 was the only consistent predictor of better BCVA at the end of each of these stages. In stage 4, higher CRT at switch and higher CRT post AFL loading predicted better BCVA. A longer time from first presentation to switch predicted better BCVA after AFL loading whilst a longer time on RBZ PRN therapy predicted worse BCVA after AFL loading. The number of injections did not predict BCVA at any stage. Predictors of CRT included the CRT at the beginning of each stage. Higher final CRT was also predicted by higher CRT and better BCVA post AFL loading and by worse BCVA and lower CRT after RBZ loading [see Additional file 1].

\section{RBZ PRN vs. AFL PRN}

A comparable trend of disease progression was observed following PRN therapy for both RBZ and AFL. 


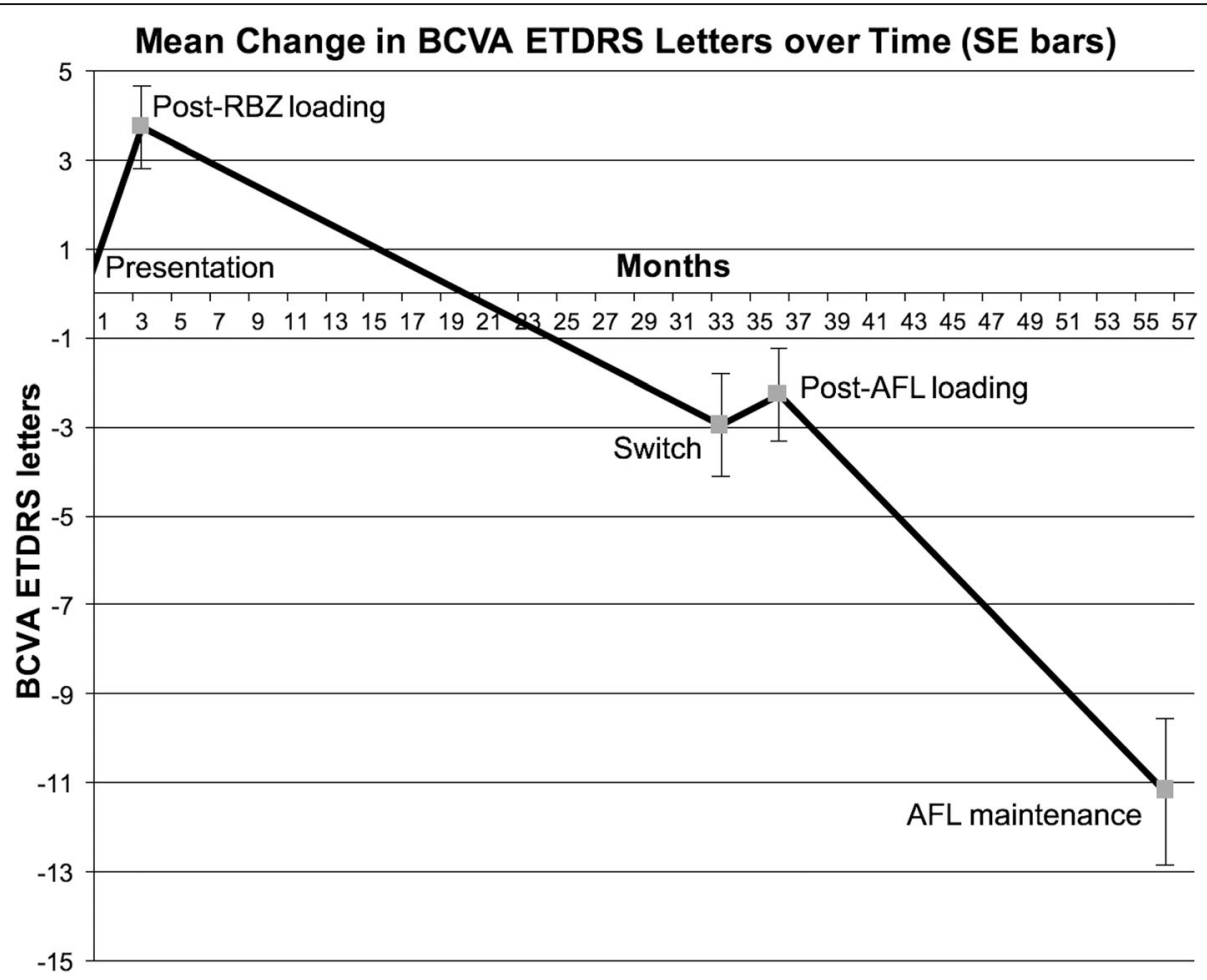

Fig. 2 Change in ETDRS letters with standard error bars

There was a mean loss of 6.7 letters $(p<0001)$ BCVA and a mean gain of $24 \mu \mathrm{m}(p<0.001)$ after RBZ PRN therapy. Similarly, there was a mean loss of 8.9 letters $(p<$ $0.001)$ BCVA and a mean gain of $12 \mu \mathrm{m}(p<0.05)$ CRT following PRN treatment with AFL PRN therapy.

In order to investigate whether response to RBZ PRN treatment correlated with AFL PRN treatment, we categorised the change in CRT and BCVA during the RBZ PRN treatment phase into different groups [see Additional file 2]. Spearman's rank-order correlation coefficient revealed no significant correlation between these different groups with respect to change in CRT or BCVA during AFL PRN, suggesting no significant link between RBZ response and AFL response in our patient cohort.

\section{Data on patients having both eyes treated}

A comparison of the data on 25 patients who had both eyes treated was made with the data from the main study. The purpose of this was to investigate if the BCVA and CRT changes across this group were similar to those in the main study. We also assessed for any

Mean Change in CRT over Time (SE bars)

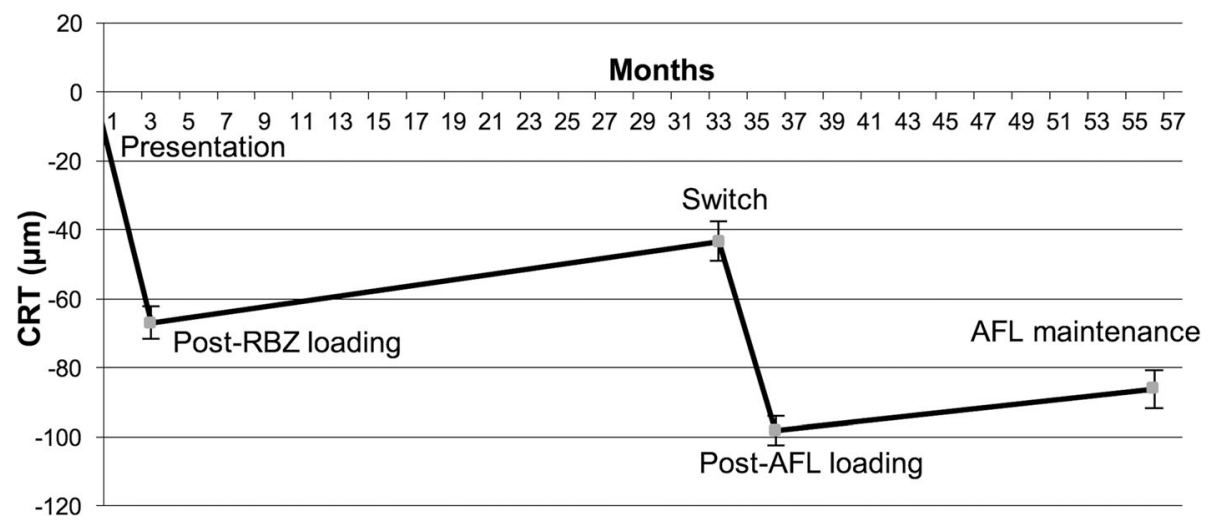

Fig. 3 Change in central retinal thickness with standard error bars 
Table 2 CRT changes following AFL PRN treatment

\begin{tabular}{llll}
\hline CRT reduced & $\mathrm{N}(\%)$ & CRT increased & $\mathrm{N}(\%)$ \\
\hline$\geq 50 \mu \mathrm{m}$ & $23(12 \%)$ & $\geq 50 \mu \mathrm{m}$ & $38(21 \%)$ \\
$\geq 100 \mu \mathrm{m}$ & $8(4 \%)$ & $\geq 100 \mu \mathrm{m}$ & $16(9 \%)$ \\
\hline
\end{tabular}

correlation between the data from the two eyes. In summary, changes in BCVA and CRT for the 'both eye' group were found to be similar to those in the main study. We found no significant correlation between the right eye and left eye data for this group [see Additional file 3].

\section{Switch-back data}

Out of 207 study eyes switched to AFL, 7 were switched back to RBZ after AFL loading due to clinical worsening of intra- or sub-retinal fluid. At switch, they had received an average of $15 \mathrm{RBZ}$ injections over a treatment period of 2.2 years. After AFL loading they had a mean loss of 3.8 ETDRS letters and mean increase of $25 \mu \mathrm{m}$ CRT. At the final data collection, these patients had been followed-up for an average of 23 months after switch. By this time, they had received an average of 4.7 AFL injections and 25 RBZ injections. Compared to post-AFL loading results they had lost an average of 4.6 ETDRS letters with no change in mean CRT.

\section{Data on patients not receiving any further IVT injections}

Following 3 AFL loading injections, 7 patients continued under regular follow-up but received no further antiVEGF injections. Prior to switch they had received an average of $6.6 \mathrm{RBZ}$ injections. At the final data collection, these patients had an average CRT of $213 \mu \mathrm{m}$ and average ETDRS score of 56 letters. Compared to results collected after AFL loading this represented a CRT reduction of $34 \mu \mathrm{m}$ and a loss of 5 ETDRS letters.

\section{Adverse events}

Adverse events were monitored during the period of AFL treatment and are outlined in Table 3. There were no cases of endophthalmitis.

\section{Discussion}

This study compared visual and anatomical outcomes in nvAMD patients who already appeared to have persistent or recurrent nvAMD on RBZ IVT and subsequently transitioned to AFL using a PRN dosing schedule after an initial loading period.

In clinical trials, AFL has been shown to have a similar efficacy and side effect profile to RBZ but with a longer dosing interval [17]. It has been suggested that AFL may be more effective in cases of refractory disease following preclinical studies which have shown that VEGF
Table 3 Adverse events reported during the study

\begin{tabular}{ll}
\hline Characteristics & Frequency \\
\hline Significant loss in VA (progressive retinal scarring) & 2 \\
Epithelial defect secondary to intravitreal injection & 1 \\
Subretinal haemorrhage & 1 \\
Subconjunctival haemorrhage & 1 \\
Retinal tear requiring retinopexy & 1 \\
Atrial fibrillation & 1 \\
Stroke & 1 \\
VZV infection/Shingles & 2 \\
Metastatic cancer diagnosed during treatment & 1 \\
\hline
\end{tabular}

Trap-Eye binds to VEGF-A with a higher affinity than other anti-VEGF molecules [18]. Additionally, it also acts on other molecules that may result in intra- or sub-retinal fluid such as placental growth factor (PIGF) which are not inhibited by other anti-VEGF drugs [18]. We found that a small number of patients $(n=7)$ did not require any further treatment after initial AFL loading. However, an equal number were also switched back to RBZ due to worsening of intraand/or sub-retinal fluid.

The outcomes in this study show that initial visual gains made by RBZ therapy on treatment-naive eyes are gradually lost over time using a PRN schedule of treatment. In our study, BCVA and CRT showed significant correlation in stage 1 but not in stages 2, 3 or 4 . This suggests that at early stages BCVA may mainly be affected by intra- or sub-retinal fluid which is reflected by CRT. However, at later stages the CRT reflects remaining neuroretinal thickness rather than intra- or sub-retinal fluid. Consequently, higher CRT should lead to better vision. This is supported by findings in stage 4 of our study which found that higher CRT post AFL loading predicted better final BCVA. We did not measure neuroretinal thickness separately in this study. Similar results of a disparity in CRT and BCVA have been found in long term studies with one anti-VEGF agent alone using PRN dosing [10, 19-21] and following transitioning between anti-VEGF agents [22].

A recent systematic review on patients with treatmentresistant nvAMD on bevacizumab or RBZ subsequently switched to AFL concluded that switching led to significant improvement in CRT but only static BCVA [23]. In contrast, we found that there was gradual deterioration in CRT and BCVA with AFL PRN therapy following an initial improvement with AFL loading. This difference in outcomes might be explained by the longer mean follow-up period (24 months) after AFL switch in our study compared to that of the studies (6-12 months) included by Spooner et al. [23] in their review. A likely interpretation is that a follow-up period of 12 months 
might not be long enough to capture the full longterm effects of switching to AFL.

There has already been some evidence that outcomes in routine clinical practice for new medications do not match the outcomes found in clinical trials where there are stringent inclusion and exclusion criteria and strict follow-up schedules [24, 25]. A potential cause of the deterioration of vision in stage 2 and the subsequent non-significant improvement of vision and significant improvement in CRT after AFL loading could be under treatment. In this study, patients were treated using a PRN protocol. However, this study compares well with recently published PRN treatment clinical trials when comparing numbers of injections per eye per year. The PrONTO study applied 9.9 injections per eye over two years using OCT guided increase in fluid as a guide to retreatment [7]. This approximates to $0.41 \mathrm{RBZ}$ injections per eye per month. A comparable 0.37 RBZ injections were performed per eye per month in this study. Additionally, we did not find that the number of injections predicted better outcomes in the analysis of this study which suggests that the patients were not significantly undertreated.

There is increasing evidence that tachyphylaxis with anti-VEGF agents may play a part in reduction of clinical efficacy with time [26, 27]. In our study, some of the effect may also be a result of tachyphylaxis to RBZ and the subsequent benefit of switching anti-VEGF agents. It is unclear whether a change to another anti-VEGF agent such as bevacizumab would also have resulted in similar short term improvements as has been reported elsewhere [28]. Tachyphylaxis to AFL treatment may also explain the progressive worsening of visual acuity and CRT following longer term treatment with AFL in stage 4 [29]. In our study only seven patients were reverted back to RBZ due to clinical failure of AFL. It would be interesting to see the effects on CRT and BCVA of switching back more patients to RBZ in a future study in order to estimate the effect of tachyphylaxis on clinical response.

A limitation of our study may be the retrospective review of notes to obtain data. The retrospective data relies on the quality of record keeping to ensure accuracy of the data and may particularly affect history, examination findings and BCVA. Nonetheless, measurements were taken at each visit and as a result an objective measure of CRT was obtained for each time point.

Long-term data comparing RBZ and AFL injection frequency has suggested a reduced required injection frequency with AFL using a treat and extend protocol [30]. However, we found that the number of injections required for treatment increased with an AFL PRN treatment regime. 0.47 AFL injections per eye per month were performed compared to $0.37 \mathrm{RBZ}$ injections per eye per month. Our study is unable to provide direct comparison of injection rates as no control group was used and AFL treatment was performed on those previously treated with RBZ and not on treatment naïve patients. Despite the increased injection rate with AFL we continued to notice a decline in visual acuity again confirming that a degenerative process may have already started prior to switch which could not be halted by AFL therapy. A future study plans to examine a costbenefit analysis of transitioning patients to AFL on a PRN basis as we found that there was an increase in the number of injections required with AFL which may negate the possible cost savings reported [22].

\section{Conclusion}

In summary, this study finds that in patients with nvAMD who are treated with PRN RBZ therapy, macular anatomy is significantly improved following AFL loading. However, this real world clinical data shows that, in the longer term, AFL does not halt the slow deterioration in BCVA and CRT which occurs in patients switched from RBZ despite a higher injection rate using PRN AFL dosing.

\section{Additional files}

Additional file 1: Supplementary data. Correlated component regression analysis examining the predictors of change in BCVA or CRT at each of the 4 stages of the study. (PDF $38 \mathrm{~kb}$ )

Additional file 2: Supplementary data. Subgroup analysis of patients who incompletely responded to RBZ based on the extent of response to RBZ PRN therapy. (PDF $23 \mathrm{~kb}$ )

Additional file 3: Supplementary data. Analysis of patients having both eyes treated. (PDF 17 kb)

\section{Abbreviations}

AFL: Aflibercept; BCVA: Best-corrected visual acuity; CRT: Central retinal thickness; EDTRS: Early Treatment Diabetic Retinopathy Study; FDA: Federal Drug Administration; IVT: Intravitreal therapy; nvAMD: Neovascular age-related macular degeneration; OCT: Optical coherence tomography; PRN: Pro re nata; RBZ: Ranibizumab; VEGF: Vascular endothelial growth factor

\section{Acknowledgements}

Previous presentations: (1) Scottish Ophthalmology Club Spring Meeting 2015 oral presentation and (2) The Association for Research in Vision and Ophthalmology (ARVO) Annual Conference 2015 poster presentation. We would like to thank Professor Lingyun Cheng, Shiley Eye Institute, University of California, San Diego, for his statistical input.

\section{Funding}

SB was supported by a Wellcome Trust STMTI scheme (grant number R42141) during the writing of this article. FE, PA, PS, CL, AA, BD and PC certify that no funding was received.

\section{Availability of data and materials}

All data generated or analysed during this study are included in this published article [and its additional files].

\section{Authors' contributions}

FE and SB contributed equally to the writing of this article and participated in data analysis and interpretation. PC devised the plan for the study and 
edited the article. PA provided statistical analysis, participated in data preparation and assisted with manuscript drafting. PS and CL participated in data collection and assisted with manuscript revision. BD and AA performed the injections in the study and examined the patients included in the study. All authors read and approved the final manuscript.

\section{Authors' information}

None

\section{Ethics approval and consent to participate}

Verbal informed consent for prospective data collection was obtained from all participants at the first injection visit by the treating clinician. The study was given ethical approval, granted a waiver of documentation of informed consent for retrospective analysis and approved for verbal consent with regard to prospective follow-up by the NHS Lothian research ethics committee with approval number 09/S1101/05. All procedures adhered to the tenets of the Declaration of Helsinki.

\section{Consent for publication}

Not applicable

\section{Competing interests}

Commercial relationship - FE (Recipient), SB (Recipient) and PC (Recipient) Bayer UK Ltd. The sponsor had no role in the design or conduct of this research. PA, PS, CL, AA, BD and PC have no competing interests to report.

\section{Publisher's Note}

Springer Nature remains neutral with regard to jurisdictional claims in published maps and institutional affiliations.

\section{Author details}

${ }^{1}$ Royal Infirmary of Edinburgh, Edinburgh, UK. ${ }^{2}$ The Princess Alexandra Eye Pavilion, Edinburgh, UK. ${ }^{3}$ Health and Environment Group, Heriot Watt University, Edinburgh, UK. ${ }^{4}$ College of Medicine \& Veterinary Medicine, University of Edinburgh, Edinburgh, UK.

Received: 4 October 2017 Accepted: 22 January 2018

Published online: 30 January 2018

\section{References}

1. Bourne RRA, Jonas JB, Flaxman SR, Keeffe J, Leasher J, Naidoo K, et al. Prevalence and causes of vision loss in high-income countries and in eastern and Central Europe : 1990 - 2010. Br J Ophthalmol. 2014;98:629-38.

2. Minassian DC, Reidy A, Lightstone A, Desai P. Modelling the prevalence of age-related macular degeneration (2010-2020) in the UK: expected impact of anti-vascular endothelial growth factor (VEGF) therapy. Br J Ophthalmol. 2011:95:1433-6

3. Gaudreault J, Fei D, Rusit J, Suboc P, Shiu V. Preclinical pharmacokinetics of ranibizumab (rhuFabV2) after a single intravitreal administration. Investig Ophthalmol Vis Sci. 2005;46:726-33.

4. Brown DM, Kaiser PK, Michels M, Soubrane G, Heier JS, Kim RY, et al. Ranibizumab versus verteporfin for neovascular age-related macular degeneration. N Engl J Med. 2006:355:1432-44.

5. Rosenfeld PJ, Brown DM, Heier JS, Boyer DS, Kaiser PK. Ranibizumab for Neovascular Age-Related Macular Degeneration. N Engl J Med. 2006; 355:1419-31.

6. Oishi A, Mandai M, Nishida A, Hata M, Matsuki T, Kurimoto Y. Remission and dropout rate of anti-VEGF therapy for age-related macular degeneration. Eur J Ophthalmol. 2011;21:777-82.

7. Lalwani GA, Rosenfeld PJ, Fung AE, Dubovy SR, Michels S, Feuer W, et al. A variable-dosing regimen with Intravitreal Ranibizumab for Neovascular agerelated macular degeneration: year 2 of the PrONTO study. Am J Ophthalmol. 2009:148:43-58.e1.

8. Holz FG, Amoaku W, Donate J, Guymer RH, Kellner U, Schlingemann RO, et al, Safety and efficacy of a flexible dosing regimen of ranibizumab in neovascular age-related macular degeneration: the SUSTAIN study. Ophthalmology. 2011; 118:663-71.

9. Spaide R. Ranibizumab according to need: a treatment for age-related macular degeneration. Am J Ophthalmol. 2007;143:679-80.
10. Rofagha S, Bhisitkul RB, Boyer DS, Sadda SR, Zhang K. Seven-year outcomes in ranibizumab-treated patients in ANCHOR, MARINA, and HORIZON: a multicenter cohort study (SEVEN-UP). Ophthalmology. 2013;120:2292-9.

11. Wulff C, Wilson H, Wiegand SJ, Rudge JS, Fraser HM. Prevention of thecal angiogenesis, antral follicular growth, and ovulation in the primate by treatment with vascular endothelial growth factor trap R1R2. Endocrinology. 2002;143:2797-807.

12. Heier JS, Brown DM, Chong V, Korobelnik JF, Kaiser PK, Nguyen QD, et al. Intravitreal aflibercept (VEGF trap-eye) in wet age-related macular degeneration Ophthalmology. 2012;119:2537-48

13. Chang AA, Li H, Broadhead GK, Hong T, Schlub TE, Wijeyakumar W, et al. Intravitreal aflibercept for treatment-resistant neovascular age-related macular degeneration. Ophthalmology. 2014;121:188-92.

14. Wykoff CC, Brown DM, Maldonado ME, Croft DE. Aflibercept treatment for patients with exudative age-related macular degeneration who were incomplete responders to multiple ranibizumab injections (TURF trial). Br J Ophthalmol. 2014;98:951 LP-955.

15. Stewart MW, Rosenfeld PJ. Predicted biological activity of intravitreal VEGF trap. Br J Ophthalmol. 2008;92:667-8.

16. Elshout M, van der Reis MI, Webers CAB, Schouten JSAG. The cost-utility of aflibercept for the treatment of age-related macular degeneration compared to bevacizumab and ranibizumab and the influence of model parameters. Graefes Arch Clin Exp Ophthalmol. 2014;252:1911-20.

17. Sarwar S, Clearfield E, Soliman MK, Sadiq MA, Baldwin AJ, Hanout M, et al. Aflibercept for neovascular age-related macular degeneration. Cochrane Database Syst Rev. 2016:2:CD011346.

18. Sophie R, Akhtar A, Sepah YJ, Ibrahim M, Bittencourt M, Do DV, et al. Aflibercept: a potent vascular endothelial growth factor antagonist for Neovascular age-related macular degeneration and other retinal vascular diseases. Biol Ther. 2012;2:1-22.

19. Silva R, Axer-Siegel R, Eldem B, Guymer R, Kirchhof B, Papp A, et al. The SECURE study: long-term safety of ranibizumab $0.5 \mathrm{mg}$ in neovascular age-related macular degeneration. Ophthalmology. 2013;120:130-9.

20. Krüger Falk M, Kemp H, Sorensen TL. Four-year treatment results of neovascular age-related macular degeneration with ranibizumab and causes for discontinuation of treatment. Am J Ophthalmol. 2013;155:89-95.

21. Group WC for the UA-RMDEU. The neovascular age-related macular degeneration database: multicenter study of 92976 ranibizumab injections: report 1: visual acuity manuscript no. 2013-568. Ophthalmology. 2014;121: 1092-101.

22. Messenger WB, Campbell JP, Faridi A, Shippey L, Bailey ST, Lauer AK, et al. Injection frequency and anatomic outcomes 1 year following conversion to aflibercept in patients with neovascular age-related macular degeneration. Br J Ophthalmol. 2014:98:1205-7.

23. Spooner K, Hong T, Wijeyakumar W, Chang AA. Switching to aflibercept among patients with treatment-resistant neovascular age-related macular degeneration: a systematic review with meta analysis. Clinl Ophthalmol. 2017;11:161-77.

24. Davidson MH. Differences Between clinical trial efficacy and real-world effectiveness. Am J Manag Care. 2006:12(15 Suppl):S405-11.

25. Martin K, Bégaud B, Latry P, Miremont-Salam G, Fourrier A, Moore N. Differences between clinical trials and postmarketing use. Br J Clin Pharmacol. 2004:57:86-92.

26. Doguizi S, Ozdek S, Yuksel S. Tachyphylaxis during ranibizumab treatment of exudative age-related macular degeneration. Int J Ophthalmol. 2015;8:846-8.

27. Eghøj MS, Sørensen TL. Tachyphylaxis during treatment of exudative agerelated macular degeneration with ranibizumab. Br J Ophthalmol. 2011;96: 21 LP-23.

28. Waizel M, Rickmann A, Blanke BR, Wolf K, Kazerounian S, Szurman P. Response to bevacizumab after treatment with aflibercept in eyes with neovascular AMD. Eur J Ophthalmol. 2016;26:469-72.

29. Slean GR, Hemarat K, Khurana RN, Stewart JM. Conversion back to bevacizumab or ranibizumab for recurrent neovascular activity with aflibercept in age-related macular degeneration: a case series. Int J Retin Vitr. 2016;2·2

30. Homer N, Grewal DS, Mirza RG, Lyon AT, Gill MK. Transitioning to intravitreal aflibercept following a previous treat-and-extend dosing regimen in neovascular age-related macular degeneration: 24-month results. Eye (Lond). 2015:29:1152-5. 